\title{
Treatment deviating from guidelines does not influence status epilepticus prognosis
}

\author{
Andrea O. Rossetti $\cdot$ Vincent Alvarez . \\ Jean-Marie Januel $\cdot$ Bernard Burnand
}

Received: 25 June 2012/Revised: 26 July 2012/Accepted: 27 July 2012/Published online: 17 August 2012

(C) Springer-Verlag 2012

\begin{abstract}
Status epilepticus (SE) prognosis is related to nonmodifiable factors (age, etiology), but the exact role of drug treatment is unclear. This study was undertaken to address the prognostic role of treatment adherence to guidelines (TAG). We prospectively studied over 26 months a cohort of adults with incident SE (excluding postanoxic). TAG was assessed in terms of drug doses ( $\pm 30 \%$ of recommendations) and medication sequence; its prognostic impact on mortality and return to baseline conditions was adjusted for etiology, SE severity [Status Epilepticus Severity Score (STESS)], and comorbidities. Of 225 patients, 26 (12\%) died and 82 (36\%) were discharged with a new handicap; TAG was observed in 142 (63\%). On univariate analysis, age, etiology, SE severity, and comorbidities were significantly related to outcome, while TAG was associated with neither outcome nor likelihood of SE control. Logistic regression for mortality identified etiology [odds ratio (OR) 18.8, $95 \%$ confidence interval (CI) 4.3-82.8] and SE severity (STESS $\geq 3$; OR $1.7,95 \%$ CI 1.2-2.4) as independent predictors, and for lack of return to baseline, again etiology (OR 7.4, $95 \% \mathrm{CI}$ 3.9-14.0) and STESS $\geq 3$ (OR 1.7, $95 \%$ CI 1.4-2.2).
\end{abstract}

\footnotetext{
A. O. Rossetti ( $\square)$

Service de Neurologie, CHUV, BH-07,

1011 Lausanne, Switzerland

e-mail: andrea.rossetti@chuv.ch
}

A. O. Rossetti · V. Alvarez

Department of Clinical Neurosciences, Centre Hospitalier

Universitaire Vaudois and University of Lausanne,

Lausanne, Switzerland

J.-M. Januel · B. Burnand

Institute of Social and Preventive Medicine, Centre Hospitalier

Universitaire Vaudois and University of Lausanne,

Lausanne, Switzerland
Similar results were found for the subgroup of 116 patients with generalized-convulsive SE. Receiver operator characteristic (ROC) analyses confirmed that TAG did not improve outcome prediction. This study of a large SE cohort suggests that treatment adherence to recommendations using current medications seems to play a negligible prognostic role (class III), confirming the importance of the biological background. Awaiting further treatment trials, it appears mandatory to apply resources towards identification of new therapeutic approaches.

Keywords Outcome $\cdot$ Mortality $\cdot$ Predictor $\cdot$ Cohort

\section{Introduction}

Status epilepticus (SE) represents, after stroke, the most common neurological emergency, with a significant risk of mortality and morbidity [15, 21]. Several independent studies identified some clinical prognostic predictors, especially acute or potentially fatal etiology, advanced age, de novo presentation, and severe consciousness impairment before treatment $[10,14,26,35]$. These nonmodifiable variables reflect the biological background and the extent of brain damage, as opposed to drug treatment; however, the specific prognostic role of SE treatment quality has surprisingly received scarce attention to date. Some studies reported worse outcome after SE if treatment did not follow guidelines [3, 31, 37], but others did not [20, 30].

Given the aforementioned divergent results, and in view of the consistent large effort invested by several national societies and international organizations in producing treatment recommendations for SE, despite a disappointing paucity of good evidence apart from for first-line therapy 
[9, 18, 29, 33, 34], it appears important to better estimate the impact of SE treatment quality on patient prognosis.

\section{Patients and methods}

\section{Patients}

We analyzed our prospective registry that includes consecutive patients older than 16 years with incident SE admitted to our center, enrolled during the period 1 April 2008 to 31 May 2011 (26 months). Further details on our clinical cohort have been published elsewhere [22]. Briefly, SE was defined as continuous occurrence of seizures during more than $5 \mathrm{~min}$, as suggested by the operational definition [16], or repeated epileptic seizures without baseline recovery in between. Seizures were diagnosed clinically, but electroencephalography (EEG) confirmation was required for nonconvulsive episodes. SE episodes were identified by neurological consultants at the emergency and intensive care units, and by EEG staff. Subjects with postanoxic SE were excluded. This study was approved by our Ethic Commission.

\section{Variables}

Demographical and clinical data included age, gender, history of previous seizures, worst seizure type (partial versus generalized), level of consciousness before treatment (alert or somnolent, versus stuporous or comatose), and SE etiology; all were recorded prospectively. A validated SE clinical severity score [28] (STESS; including age, previous seizures, seizure type, and consciousness) was calculated upon admission according to the situation before treatment start (0-6 points), and categorized as $\geq 3$ (poor outcome prediction) versus $<3$ (good outcome prediction). Etiology was considered as "potentially fatal" if potentially leading per se to death if not specifically treated, as detailed in previous works [22, 26]; this classification seems to account for SE prognosis better than the International League Against Epilepsy (ILAE) classification $[1,22,26]$.

Detailed medication administration to treat SE was also collected prospectively. This included drug type, administration route, loading dosage per body weight, and sequence of administration. The exact timing of administration was available only for the first medication and was dichotomized at $1 \mathrm{~h}$ following the beginning of the SE episode. SE treatment was compared with the in-house protocol in use since 2007, which is in agreement with the 2005 Swiss guidelines [13]. The recommendation includes the following intravenous treatment administrations: as first line, a slow bolus of lorazepam $0.1 \mathrm{mg} / \mathrm{kg}$, clonazepam $0.015 \mathrm{mg} / \mathrm{kg}$, or midazolam $0.2 \mathrm{mg} / \mathrm{kg}$; as second line, phenytoin $20 \mathrm{mg} / \mathrm{kg}$, or valproate $20 \mathrm{mg} / \mathrm{kg}$, or levetiracetam $20 \mathrm{mg} / \mathrm{kg}$; as third line, propofol $2 \mathrm{mg} / \mathrm{kg}$ followed by $2-10 \mathrm{mg} / \mathrm{kg} / \mathrm{h}$, or midazolam $0.2 \mathrm{mg} / \mathrm{kg}$ followed by $0.2-0.6 \mathrm{mg} / \mathrm{kg} / \mathrm{h}$, or thiopental $2 \mathrm{mg} / \mathrm{kg}$ followed by $2-5 \mathrm{mg} / \mathrm{kg} / \mathrm{h}$ (anesthetics are titrated aiming at a burstsuppression EEG with $10 \mathrm{~s}$ interburst interval). Treatment was considered "adherent" if within a $\pm 30 \%$ range of recommended dosage; the two following exceptions were accepted: (a) more generous benzodiazepine loading doses in patients with delirium tremens, (b) second-line administration of the usual antiepileptic drug (other than the aforementioned compounds) if suspected to be withdrawn in patients with epilepsy. Treatment was considered "nonadherent" if the dosage of any drug was (a) lower (undertreatment) or (b) greater (overtreatment) than the $30 \%$ range, or (c) was wrong, i.e., the treatment sequence was not respected (e.g., phenytoin as first line; anesthetic agent given together with the second-line drug in patients with simple-partial, complex-partial, or myoclonic SE without respiratory or cardiovascular impairment).

The Charlson Comorbidity Index (CCI), a validated composite score of 19 different medical conditions, was used to quantify active medical conditions preceding SE (apart from its etiology), weighted by the risk of 1-year mortality [4]. CCI was calculated after hospital discharge, from the computerized medical files, and categorized into three groups: $\mathrm{CCI}=0, \mathrm{CCI}=1-2$, and $\mathrm{CCI}=3-19$. Outcome at hospital discharge represented the primary outcome; it was prospectively categorized into three, mutually exclusive items: return to baseline (premorbid functional and neurological) status, new disability, or death.

\section{Statistical analyses}

Each potential predictor of the SE outcomes "lack of return to baseline" (i.e., mortality or new disability) and "mortality" was analyzed individually for its relationship with each outcome, using $\chi^{2}$ or two-sided Fisher exact tests when appropriate. Stepwise logistic regressions were performed to analyze the role of potential predictors, using etiology, SE severity (STESS score, which includes age), medical comorbitity, and treatment adherence. Goodness of fit was assessed using $\chi^{2}$ tests. We calculated receiver operator characteristic (ROC) curves for the different models, using a nonparametric approach and applying Bonferroni corrections to obtain a global $P<0.05$ in case of multiple comparisons; for this analysis, every medical condition included in the CCI was also considered as an individual variable. Calculations were performed using Stata software (version 9; College Station, TX). 


\section{Results}

Among 263 SE episodes recorded during the study period, we identified $225(86 \%)$ incident patients. Mean patient age was $61.1( \pm 18.4)$ years, and $102(45 \%)$ were women; $93(41 \%)$ had a known epilepsy diagnosis, and 97 (43\%) experienced a potentially fatal etiology. SE lasted between 5 and $29 \mathrm{~min}$ before diagnosis in $27(12 \%)$ subjects. Regarding semiology, 32 (14\%) experienced simple-partial, $71(32 \%)$ complex-partial, $106(47 \%)$ generalizedconvulsive, $10(4 \%)$ subtle, $5(2 \%)$ absence, and 1 myoclonic (genetic generalized epilepsy) SE forms; 134 (56\%) had a severe consciousness impairment before treatment, defined as stupor (arousal without response) or coma. Treatment was delayed for more than $1 \mathrm{~h}$ after the beginning of SE in 139 (62\%) patients. In this subgroup, the prevalence of generalized-convulsive SE was clearly lower than in patients treated within $1 \mathrm{~h}(54 / 139$ versus $\left.62 / 86, P<0.001, \chi^{2}\right)$. Treatment was judged not adherent to guidelines in 83 (37\%) patients, while in 6 patients treatment adherence could not be assessed 11 patient died before treatment institution, while in another 5 (who returned to baseline) the SE episode stopped spontaneously]; for further analyses, these subjects were added to the "adherent" group.

Regarding outcome, 26 (12\%) patients died and 82 (36\%) left our hospital with a new disability. Demographics and relevant clinical variables of the studied cohort, stratified according to functional outcome at hospital discharge, are illustrated in Table 1. While age, etiology, SE severity (STESS score), and comorbidity were significantly related to outcome, treatment latency and adherence were not. Considering the subgroup of 116 patients with generalized-convulsive and nonconvulsive SE in coma, representing the most severe forms of SE, lack of treatment adherence to guidelines was again not related to mortality (5/12 patients with fatal outcome were treated violating guidelines versus $31 / 104$ surviving, $P=0.51$, Fisher) or return to baseline conditions (19/50 not returning to baseline received treatment not adherent to guidelines versus $17 / 66$ returning to baseline, $P=0.16, \chi^{2}$ ). Table 2 details the reasons for nonadherent treatment according to outcome: no statistical difference emerged, even if the proportion of nonadherent treatment was lowest in patients with the most favorable outcome (i.e., return to baseline). Table 3 shows treatment adherence according to several clinical and demographical variables. Greater latency to treatment institution was related to any violation in the treatment protocol, and patients with a potentially fatal etiology tended to be treated more often with high dosages. Even if nonsignificant, higher age tended somewhat to be related to medication underdosage (especially benzodiazepines, data not shown), while patients with greater severity (STESS) were somewhat more likely to receive high drug dosages. Finally, in 13 patients (6\% of the total, all with fatal outcome) the SE episode was not controlled; the distribution of noncontrolled episodes among adherent and nonadherent treatment groups resulted similar.

Logistic regression modeling using mortality as the dependent variable identified potentially fatal etiology (OR $18.8,95 \%$ CI 4.3-82.8) and STESS $\geq 3$ (OR 1.7, $95 \%$ CI 1.2-2.4) as independent predictors, while treatment adherence (dichotomous) and CCI were not. As regards lack of return to baseline clinical conditions, results were similar: potentially fatal etiology (OR 7.4, $95 \%$ CI 3.9-14.0) and STESS $\geq 3$ (OR 1.7, $95 \%$ CI 1.4-2.2) were independently related to this outcome, as opposed to treatment adherence and CCI. All models showed acceptable goodness of fit, apart from those including CCI for return to baseline. To further analyze the role of treatment quality, we constructed ROC curves for the two outcomes (mortality, lack of return to baseline) using four models. The results are given in Figs. 1 and 2. While for both outcomes the prognostic accuracy (area under the curve) significantly improved after adding STESS to etiology (and, for baseline return, also adding CCI), consideration of treatment adherence did not show any notable impact on prediction. For these comparisons, significance was set at $P<0.017$ (Bonferroni).

\section{Discussion}

This study provides class III evidence that SE drug treatment, after consideration of robust predictors such as etiology, SE severity including age, and comorbidities, seems to have no major effect on prognosis even if administered violating current guidelines.

Two decades ago, a multicenter retrospective assessment on 346 patients with convulsive SE in The Netherlands showed that "insufficient" treatment was more frequent in patients who died (45\%) or had new sequelae (22\%), as compared with those having good outcome $(10 \%)$ [31]. A prospective comparison of 57 adults with SE between a rural and an urban center in Italy disclosed better prognosis in the former [37]; the authors showed that better medical management in the peripheral hospital (83 versus $73 \%$ of "appropriate" treatment) was strongly and independently related to clinical outcome (OR 21.09). A subsequent prospective study by our group comparing treatment appropriateness in 54 adult patients between our tertiary referral center and peripheral hospitals in our region did not support that hypothesis, since the functional prognosis of subjects treated in the referral center was not different, despite significantly better application of SE treatment guidelines $(97 \%$ in the center, $78 \%$ in 
Table 1 Demographics and clinical data of incident patients with SE according to outcome

$S E$ status epilepticus, $C C I$ Charlson Comorbidity Index, STESS Status Epilepticus Severity Score

Table 2 Comparison of reasons for SE treatment not adherent to guidelines according to SE outcome

$S E$ status epilepticus

Table 3 Comparison of drug SE treatment appropriateness stratified by demographics and clinical factors

$S E$ status epilepticus, $C C I$

Charlson Comorbidity Index, STESS Status Epilepticus Severity Score

${ }^{\text {a }}$ Of the remaining patients, six could not be assessed as regards their treatment adherence to guidelines; 83 were treated violating the guidelines

\begin{tabular}{|c|c|c|c|c|c|}
\hline & $\begin{array}{l}\text { Mortality } \\
(\%)\end{array}$ & $\begin{array}{l}\text { New disability } \\
(\%)\end{array}$ & $\begin{array}{l}\text { Return to baseline } \\
(\%)\end{array}$ & $P$ value & Test \\
\hline Total & $\begin{array}{r}26 / 225 \\
(11.6)\end{array}$ & $82 / 225(36.4)$ & $117 / 225(52.0)$ & & \\
\hline Age $\geq 65$ years & $\begin{array}{l}17 / 26 \\
(65.4)\end{array}$ & $53 / 82(64.6)$ & $34 / 117$ (29.0) & $<0.001$ & $\chi^{2}$ \\
\hline Potentially fatal etiology & $\begin{array}{l}24 / 26 \\
(92.3)\end{array}$ & 48/82 (58.5) & 25/117 (21.4) & $<0.001$ & $\chi^{2}$ \\
\hline Treatment latency $>1 \mathrm{~h}$ & $\begin{array}{l}15 / 26 \\
(57.7)\end{array}$ & $53 / 82(64.6)$ & $71 / 117(60.4)$ & 0.769 & $\chi^{2}$ \\
\hline STESS $\geq 3$ & $\begin{array}{l}21 / 26 \\
(80.8)\end{array}$ & $58 / 82(70.7)$ & $51 / 117(43.6)$ & $<0.001$ & $\chi^{2}$ \\
\hline \multicolumn{6}{|l|}{$\mathrm{CCI}$} \\
\hline 0 & $3 / 26(11.6)$ & $24 / 82(29.3)$ & 45/117 (38.5) & $<0.001$ & Fisher \\
\hline $1-2$ & $3 / 26(11.6)$ & $28 / 82(34.1)$ & $39 / 117(33.3)$ & & \\
\hline 3 & $\begin{array}{l}20 / 26 \\
(76.9)\end{array}$ & $30 / 82(36.6)$ & $33 / 117(28.2)$ & & \\
\hline $\begin{array}{l}\text { Treatment not adherent to } \\
\text { guidelines }\end{array}$ & $\begin{array}{l}13 / 26 \\
(50.0)\end{array}$ & $33 / 82(40.2)$ & $37 / 117(32)$ & 0.157 & $\chi^{2}$ \\
\hline
\end{tabular}

\begin{tabular}{llllll}
\hline Treatment & Mortality $(\%)$ & New disability $(\%)$ & Return to baseline $(\%)$ & $P$ value & Test \\
\hline Overdosed & $2 / 26(7.7)$ & $3 / 82(3.7)$ & $4 / 117(3.4)$ & 0.542 & Fisher \\
Underdosed & $7 / 26(26.9)$ & $20 / 82(24.4)$ & $23 / 117(19.7)$ & 0.579 & Fisher \\
Wrong sequence & $6 / 26(23.1)$ & $17 / 82(20.7)$ & $14 / 117(12)$ & 0.153 & Fisher \\
\hline
\end{tabular}

\begin{tabular}{|c|c|c|c|c|c|c|}
\hline & $\begin{array}{l}\text { Treatment adherent } \\
\text { to guidelines }{ }^{\mathrm{a}}(\%)\end{array}$ & $\begin{array}{l}\text { Overdosed } \\
\text { treatment } \\
(\%)\end{array}$ & $\begin{array}{l}\text { Underdosed } \\
\text { treatment }(\%)\end{array}$ & $\begin{array}{l}\text { Wrong } \\
\text { treatment } \\
\text { sequence }(\%)\end{array}$ & $P$ value & Test \\
\hline Total & $136 / 225(60.4)$ & $9 / 225(4.0)$ & $50 / 225(22.2)$ & $37 / 225(16.4)$ & & \\
\hline $\begin{array}{l}\text { Age } \\
\geq 65 \text { years }\end{array}$ & $56 / 136(41.2)$ & $4 / 9(44.4)$ & $31 / 50(62.0)$ & $21 / 37(56.8)$ & 0.279 & Fisher \\
\hline $\begin{array}{l}\text { Potentially } \\
\text { fatal } \\
\text { etiology }\end{array}$ & $53 / 136(39.0)$ & 7/9 (77.7) & $23 / 50(46.0)$ & $19 / 37(51.4)$ & 0.091 & Fisher \\
\hline $\begin{array}{l}\text { Treatment } \\
\text { latency } \\
>1 \mathrm{~h}\end{array}$ & $69 / 136(50.7)$ & 7/9 (77.7) & $39 / 50(78.0)$ & $32 / 37(85.6)$ & $<0.001$ & Fisher \\
\hline $\begin{array}{l}\text { STESS } \geq 3 \\
\text { CCI }\end{array}$ & $79 / 136(56.8)$ & $8 / 9$ (88.8) & $31 / 50(62.0)$ & $20 / 37(54.1)$ & 0.273 & Fisher \\
\hline 0 & $44 / 136(32.4)$ & $1 / 9(11.1)$ & $15 / 50(30.0)$ & $12 / 37(32.4)$ & 0.431 & Fisher \\
\hline $1-2$ & $39 / 136(28.6)$ & $3 / 9(33.3)$ & $15 / 50(30.0)$ & $16 / 37(43.2)$ & & \\
\hline 3 & $53 / 136(39.0)$ & $5 / 9(55.5)$ & $20 / 50(40.0)$ & $9 / 37(24.3)$ & & \\
\hline $\begin{array}{l}\text { SE not } \\
\text { controlled }\end{array}$ & $7 / 136(5)$ & $1 / 9(11)$ & $3 / 50(6)$ & 4/37 (11) & 0.417 & Fisher \\
\hline
\end{tabular}

peripheral settings) [30]. This is in line with a retrospective Canadian analysis on 45 adults with convulsive SE: while $71 \%$ of them received appropriate treatment, their clinical outcome was similar, apart from SE length [20]. Most recently, another prospective study carried out in France, including 101 adults with generalized-convulsive SE, identified treatment adherence to guidelines as a powerful and independent predictor of seizure control: initial treatment according to local recommendations had a $74 \%$ efficacy rate versus $29 \%$ in cases with protocol violations 
Fig. 1 Assessment of different models for the prediction of mortality in 225 patients with incident status epilepticus

\begin{tabular}{|l|c|c|}
\hline & Area & 95\% confidence interval \\
\hline $\begin{array}{l}\text { Model 1 } \\
\text { Potentially fatal etiology }\end{array}$ & 0.78 & $0.71-0.84$ \\
\hline $\begin{array}{l}\text { Model 2 } \\
\text { Potentially fatal etiology, STESS }\end{array}$ & 0.84 & $0.78-0.91$ \\
\hline $\begin{array}{l}\text { Model 3 } \\
\text { Potentially fatal etiology, STESS, CCI }\end{array}$ & 0.91 & $0.86-0.96$ \\
\hline $\begin{array}{l}\text { Model 4 } \\
\text { Potentially fatal etiology, STESS, CCI, treatment appropriateness }\end{array}$ & 0.91 & $0.87-0.97$ \\
\hline
\end{tabular}

$\mathrm{P}=0.002$

CCI: Charlson comorbidity index; STESS= status epilepticus severity score.

\begin{tabular}{|l|l|}
\hline Model 1 vs. Model 2 & $\mathrm{P}=0.121$ \\
\hline Model 2 vs. Model 3 & $\mathrm{P}=0.005$ \\
\hline Model 3 vs. Model 4 & $\mathrm{P}=0.259$ \\
\hline
\end{tabular}

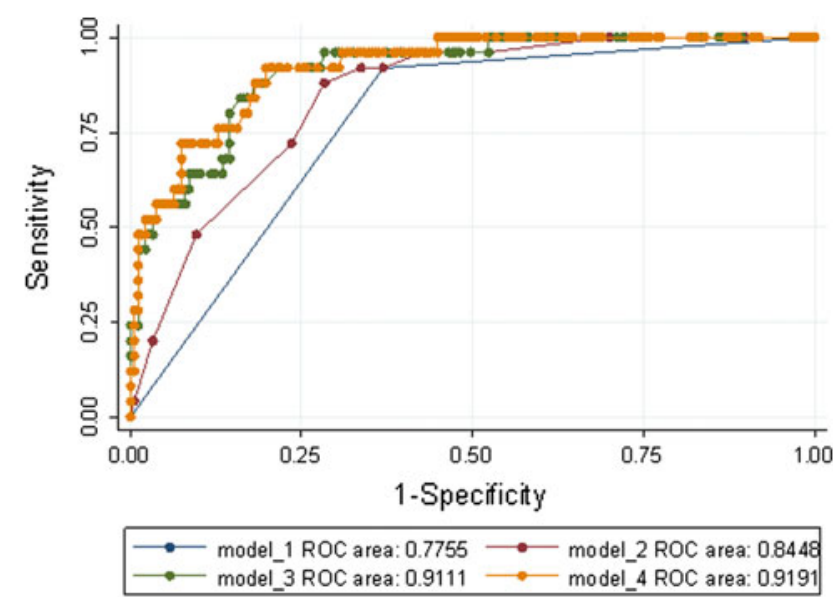

[3]. The various results of these studies probably reflect different SE definitions, patient selections, etiologies, treatment protocols, definitions of treatment appropriateness, and the chosen outcome; For instance, the French study [3] was limited to convulsive SE and its protocol was rather peculiar as compared with the French guidelines [23]; furthermore, participating physicians received specific training before the study, criteria regarding treatment appropriateness were not detailed, and data on clinical outcome were not described. Most importantly, in all mentioned studies the impact of treatment was not adjusted for other known SE outcome predictors.

Our prospective analysis, which includes more patients than the three previous prospective studies taken together $[3,30,37]$ and is not limited to generalized-convulsive SE, suggests that adherence of specific SE drug treatment to recommendations plays a relatively negligible prognostic role. This underscores the robustness of other predictors, independently identified by several groups over the last two decades. Increasing age and acute (or potentially fatal) etiology seem the most important variables $[10,14,26$, 35]; the extent of consciousness impairment and seizure type also play an independent role in some studies [26, 32], while the delay to treatment start has yielded divergent results, possibly owing to its relationship with seizure type (complex-partial or absence SE may last for a relatively long time without major sequelae), or the loss of predictive performance after the first few hours [7, 14, 26, 35]. Concomitant medical conditions may also be related to prognosis $[2,12]$. We therefore designed our analysis to be 
Fig. 2 Assessment of different models for the prediction of lack of return to baseline clinical conditions in 225 patients with incident status epilepticus

\begin{tabular}{|l|c|c|}
\hline & Area & 95\% confidence interval \\
\hline $\begin{array}{l}\text { Model 11 } \\
\text { Potentially fatal etiology }\end{array}$ & 0.72 & $0.66-0.77$ \\
\hline $\begin{array}{l}\text { Model 12 } \\
\text { Potentially fatal etiology, STESS }\end{array}$ & 0.80 & $0.74-0.86$ \\
\hline $\begin{array}{l}\text { Model 13 } \\
\text { Potentially fatal etiology, STESS, CCI }\end{array}$ & 0.85 & $0.79-0.90$ \\
\hline $\begin{array}{l}\text { Model 14 } \\
\text { Potentially fatal etiology, STESS, CCI, treatment appropriateness }\end{array}$ & 0.85 & $0.80-0.90$ \\
\hline
\end{tabular}

$\mathrm{P}<0.001$

CCI: Charlson comorbidity index; STESS= status epilepticus severity score.

\begin{tabular}{|l|l|}
\hline Model 11 vs. Model 12 & $\mathrm{P}<0.001$ \\
\hline Model 12 vs. Model 13 & $\mathrm{P}=0.011$ \\
\hline Model 13 vs. Model 14 & $\mathrm{P}=0.749$ \\
\hline
\end{tabular}

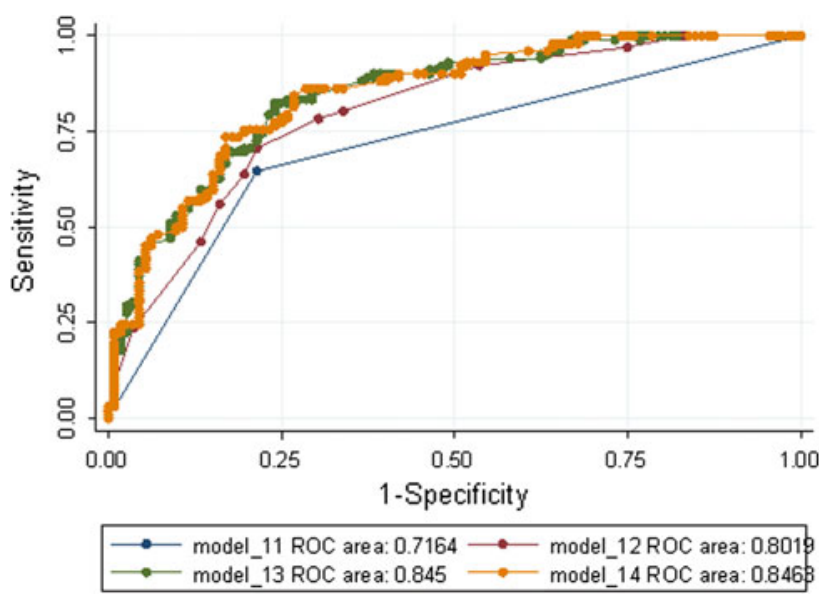

adjusted for the most important predictors: etiology, the validated STESS score, and comorbitities.

We did not find a significant prognostic role of SE drug treatment quality on outcome; this may reflect the fact that antiepileptic compounds administered in this clinical setting are exclusively "symptomatic," and that an etiological SE treatment proves often difficult (either the etiology is not know or it is not responsive to treatments, e.g., in some autoimmune forms or in devastating brain injuries). We cannot formally exclude that an effect of treatment quality might be observed in a much larger cohort; however, our results indicate that this role would be rather marginal. Nevertheless, based on our analyses, we believe that further studies of the role of a wrong medication sequence on prognosis, which seems to bear a somewhat greater risk of poor outcome as compared with over- or underdosage, should be conducted. We observed that treatment latency $>1 \mathrm{~h}$ was significantly related to underdosage and incorrect drug sequence. This association possibly illustrates that, in presence of SE forms other than generalized convulsions, longer treatment delay and reduced adherence to guidelines may coexist because of a perceived lower risk of sequelae. Conversely, with potentially fatal SE etiologies and higher STESS score, slightly more patients received overdosed drugs, possibly reflecting a propensity for more aggressive treatments in severe cases.

This observational study reflects practice in our center: at the beginning, patients are mostly treated by paramedics, emergency physicians, and/or consultant neurologists. Epileptologists are involved only after SE is suspected (and during working days). Protocol "violations" occurred despite the fact that every consultant neurologist and 
emergency physician is periodically informed on the treatment flow for patients with SE, and might in part also reflect particular clinical situations (such as the use of low benzodiazepine doses in elderly subjects). We believe that this is similar to most centers.

This study has some limitations. First, it is based on Swiss SE treatment guidelines [13]; however, apart from clonazepam, which is popular in French-speaking countries [23], this treatment protocol is extremely similar to other current national and international guidelines [9, 18, 19, 33, 36]. Second, the definition of appropriateness implies some arbitrary judgment; we chose a deviation of $30 \%$ in the dosage, or an alteration of the medication sequence, as we believe that these "violations" may prove of clinical importance (e.g., the load of $1 \mathrm{~g}$ phenytoin in a $75-\mathrm{kg}$ patient is mostly felt to be insufficient). While we acknowledge that this approach was not based on previous evidence, it allows uniform scoring of treatment appropriateness throughout the cohort. In this context, we emphasize that previous studies provided far less clear criteria about treatment quality [3, 20, 30, 37]. Third, our study is a clinical cohort observed in one academic setting only. However, it is characterized by a prospective design with homogeneous assessment criteria over the entire recruitment period, elements that in our view corroborate its internal validity. We also believe that the external validity of our results is warranted for similar hospitalized patients in comparable settings, as demographics and mortality are in line with most population-based studies in Europe [5, 8, 11], and, to a broader extent, North America $[6,14,38]$ (especially after exclusion of postanoxic encephalopathy [27]). Fourth, although large as compared with previous studies, our number of cases might prevent identification of small effects of treatment on prognosis; to identify a difference of $5 \%$ in return to baseline conditions (e.g., 55 versus $50 \%$ ), a sample of more than 3,000 patients would be required (power 0.8, alpha error 0.05). This would imply that at least 10 centers would recruit for over 2 years. Finally, no patient in this cohort was treated with extreme protocol violations (e.g., huge toxic doses or pseudoplacebos of any medication, as almost all violations were included in a window of 33-200 \% of the recommended doses); it nevertheless is likely that in such a setting the clinical outcome would be markedly influenced by "treatment".

Our findings, although somewhat surprising, may have an important practical impact on clinical practice. They corroborate the role of nonmodifiable clinical predictors, representing the biological background on which the SE occurs: the fact that patients with noncontrolled SE were similarly distributed among adherent and nonadherent treatment groups further suggests that, if SE is per se "treatable" according to its underlying background, it may be controlled with further treatment despite inadequate initial management. Pending well-designed drug trials in patients with SE, the present results may challenge the need of further detailed SE treatment guidelines. Protocols are certainly useful in smoothing and fastening the interplay of medical personnel involved in management of SE [9, 24, 29,33 ], and we continue to use a protocol in our institution, but it seems that identification of new specific therapeutic approaches targeting, for example, the etiology, epileptogenic mechanisms, or inflammation [17, 25], is urgently needed, since the role of medical treatment appears marginal using the current antiepileptic drug arsenal.

Conflicts of interest A.O.R. received research grants from Pfizer, UCB, GSK, and Sandoz. All other authors do not have anything to disclose.

Ethical standard This study has been approved by the appropriate ethics committee and has therefore been performed in accordance with the ethical standards laid down in the 1964 Declaration of Helsinki.

\section{References}

1. Commission on Epidemiology and Prognosis, International League Against Epilepsy (1993) Guidelines for epidemiologic studies on epilepsy. Epilepsia 34:592-596

2. Alvarez V, Januel JM, Burnand B, Rossetti AO (2012) Role of comorbidities in outcome prediction after status epilepticus. Epilepsia 53:e89-e92

3. Aranda A, Foucart G, Ducasse JL, Grolleau S, McGonigal A, Valton L (2010) Generalized-convulsive status epilepticus management in adults: a cohort study with evaluation of professional practice. Epilepsia 51:2159-2167

4. Charlson ME, Pompei P, Ales KL, MacKenzie CR (1987) A new method of classifying prognostic comorbidity in longitudinal studies: development and validation. J Chronic Dis 40:373-383

5. Coeytaux A, Jallon P, Galobardes B, Morabia A (2000) Incidence of status epilepticus in French-speaking Switzerland: (EPISTAR). Neurology 55:693-697

6. DeLorenzo RJ, Hauser WA, Towne AR, Boggs JG, Pellock JM, Penberthy L, Garnett L, Fortner CA, Ko D (1996) A prospective, population-based epidemiologic study of status epilepticus in Richmond, Virginia. Neurology 46:1029-1035

7. Drislane FW, Blum AS, Lopez MR, Gautam S, Schomer DL (2009) Duration of refractory status epilepticus and outcome: loss of prognostic utility after several hours. Epilepsia 50:1566-1571

8. Govoni V, Fallica E, Monetti VC, Guerzoni F, Faggioli R, Casetta I, Granieri E (2007) Incidence of Status Epilepticus in Southern Europe: a Population Study in the Health District of Ferrara, Italy. Eur Neurol 59:120-126

9. Holtkamp M (2011) Treatment strategies for refractory status epilepticus. Curr Opin Crit Care 17:94-100

10. Holtkamp M, Othman J, Buchheim K, Meierkord H (2005) Predictors and prognosis of refractory status epilepticus treated in a neurological intensive care unit. J Neurol Neurosurg Psychiatry 76:534-539

11. Knake S, Rosenow F, Vescovi M, Oertel WH, Mueller HH, Wirbatz A, Katsarou N, Hamer HM (2001) Incidence of status epilepticus in adults in Germany: a prospective, population-based study. Epilepsia 42:714-718 
12. Koubeissi M, Alshekhlee A (2007) In-hospital mortality of generalized-convulsive status epilepticus: a large US sample. Neurology 69:886-893

13. Leppert D, Stoeckli HR, Fuhr P (2005) Directives pour le traitement de l'état de mal épileptique. Schweizerische Aerztezeitung 86. www.saez.ch/status_epilepticus_f.pdf

14. Logroscino G, Hesdorffer DC, Cascino G, Annegers JF, Hauser WA (1997) Short-term mortality after a first episode of status epilepticus. Epilepsia 38:1344-1349

15. Lowenstein DH, Alldredge BK (1998) Status epilepticus. N Engl J Med 338:970-976

16. Lowenstein DH, Bleck T, Macdonald RL (1999) It's time to revise the definition of status epilepticus. Epilepsia 40:120-122

17. Marchi N, Granata T, Freri E, Ciusani E, Ragona F, Puvenna V, Teng Q, Alexopolous A, Janigro D (2011) Efficacy of antiinflammatory therapy in a model of acute seizures and in a population of pediatric drug resistant epileptics. PLoS ONE 6:e18200

18. Meierkord H, Boon P, Engelsen B, Gocke K, Shorvon S, Tinuper $P$, Holtkamp M (2010) EFNS guideline on the management of status epilepticus in adults. Eur J Neurol 17:348-355

19. Minicucci F, Muscas G, Perucca E, Capovilla G, Vigevano F, Tinuper P (2006) Treatment of status epilepticus in adults: guidelines of the Italian League against Epilepsy. Epilepsia 47(Suppl 5):9-15

20. Muayqil T, Rowe BH, Ahmed SN (2007) Treatment adherence and outcomes in the management of convulsive status epilepticus in the emergency room. Epileptic Disord 9:43-50

21. Neligan A, Shorvon SD (2011) Prognostic factors, morbidity and mortality in tonic-clonic status epilepticus: a review. Epilepsy Res 93:1-10

22. Novy J, Logroscino G, Rossetti AO (2010) Refractory status epilepticus: a prospective observational study. Epilepsia 51:251-256

23. Outin H, Blanc T, Vinatier I (2009) Emergency and intensive care unit management of status epilepticus in adult patients and children (new-born excluded). Societe de reanimation de langue francaise experts recommendations. Rev Neurol (Paris) 165:297-305

24. Rabinstein AA (2010) Management of status epilepticus in adults. Neurol Clin 28:853-862

25. Ravizza T, Balosso S, Vezzani A (2011) Inflammation and prevention of epileptogenesis. Neurosci Lett 497:223-230
26. Rossetti AO, Hurwitz S, Logroscino G, Bromfield EB (2006) Prognosis of status epilepticus: role of aetiology, age, and consciousness impairment at presentation. J Neurol Neurosurg Psychiatry 77:611-615

27. Rossetti AO, Logroscino G, Bromfield EB (2005) Refractory status epilepticus: effect of treatment aggressiveness on prognosis. Arch Neurol 62:1698-1702

28. Rossetti, AO, Logroscino G, Milligan TA, Michaelides C, Ruffieux C, Bromfield EB (2008) Status Epilepticus Severity Score (STESS): a tool to orient early treatment strategy. J Neurol 255:1561-1566

29. Rossetti AO, Lowenstein DH (2011) Management of refractory status epilepticus in adults: still more questions than answers. Lancet Neurol 10:922-930

30. Rossetti AO, Novy J, Ruffieux C, Olivier P, Foletti GB, Hayoz D, Burnand B, Logroscino G (2009) Management and prognosis of status epilepticus according to hospital setting: a prospective study. Swiss Med Wkly 139:719-723

31. Scholtes FB, Renier WO, Meinardi H (1994) Generalized-convulsive status epilepticus: causes, therapy, and outcome in 346 patients. Epilepsia 35:1104-1112

32. Shneker BF, Fountain NB (2003) Assessment of acute morbidity and mortality in non-convulsive status epilepticus. Neurology 61:1066-1073

33. Shorvon S (2011) The treatment of status epilepticus. Curr Opin Neurol 24:165-170

34. Silbergleit R, Durkalski V, Lowenstein D, Conwit R, Pancioli A, Palesch Y, Barsan W (2012) Intramuscular versus intravenous therapy for prehospital status epilepticus. $\mathrm{N}$ Engl J Med 366:591-600

35. Towne AR, Pellock JM, Ko D, DeLorenzo RJ (1994) Determinants of mortality in status epilepticus. Epilepsia 35:27-34

36. van Rijckevorsel K, Boon P, Hauman H, Legros B, Ossemann M, Sadzot B, Schmedding E, van Zandijcke M (2006) Standards of care for non-convulsive status epilepticus: Belgian consensus recommendations. Acta Neurol Belg 106:117-124

37. Vignatelli L, Rinaldi R, Baldin E, Tinuper P, Michelucci R, Galeotti M, de Carolis P, D'Alessandro R (2008) Impact of treatment on the short-term prognosis of status epilepticus in two population-based cohorts. J Neurol 255:197-204

38. Wu YW, Shek DW, Garcia PA, Zhao S, Johnston SC (2002) Incidence and mortality of generalized-convulsive status epilepticus in California. Neurology 58:1070-1076 